\title{
Solar driven energy conversion applications based on 3C-SiC
}

Jianwu Sun ${ }^{1, a}{ }^{*}$, Valdas Jokubavicius ${ }^{1, b}$, Lu Gao ${ }^{2, c}$, Ian Booker ${ }^{1, d}$, Mattias Jansson ${ }^{1, e}$, Xinyu Liu, ${ }^{1, f}$, Jan P. Hofmann ${ }^{2, g}$, Emiel J. M. Hensen ${ }^{2, h}$, Margareta Linnarsson ${ }^{3, \mathrm{i}}$, Peter Wellmann ${ }^{4, j}$, Iñigo Ramiro ${ }^{5, k}$, Antonio Marti ${ }^{5, l}$, Rositsa Yakimova $^{1, m}$, and Mikael Syväjärvi ${ }^{1, n}$

'Department of Physics, Chemistry and Biology (IFM), Linköping University, SE-58183, Linköping, Sweden

${ }^{2}$ Department of Chemical Engineering and Chemistry, Eindhoven University of Technology, P.O. Box 513, 5600 MB Eindhoven, Netherlands

${ }^{3}$ School of Information and Communication Technology, KTH Royal Institute of Technology, SE16440 Kista, Sweden

${ }^{4}$ Department of Materials Science 6, University of Erlangen-Nuremberg, Martensstr. 7, 91058 Erlangen, Germany

${ }^{5}$ Instituto de Energía Solar, Universidad Politécnica de Madrid, E.T.S.I. Telecomunicación, Av. de la Complutense 30, 28040 Madrid, Spain

ajiasu@ifm.liu.se,bvaljo@ifm.liu.se, 9.gao@tue.nl, dianbo@ifm.liu.se, ematja307@student.liu.se, fxinyu.liu@liu.se, 9j.p.Hofmann@tue.nl, he.j.m.hensen@tue.nl, 'marga@kth.se,

jpeter.wellmann@fau.de, kinigo.ramiro@ies-def.upm.es, lamarti@etsit.upm.es, mrosya@ifm.liu.se, ${ }^{n}$ misyv@ifm.liu.se

*corresponding author: Jianwu Sun

Keywords: cubic silicon carbide (3C-SiC), solar cell, photoelectrochemical (PEC) water splitting

Abstract. There is a strong and growing worldwide research on exploring renewable energy resources. Solar energy is the most abundant, inexhaustible and clean energy source, but there are profound material challenges to capture, convert and store solar energy. In this work, we explore $3 \mathrm{C}$-SiC as an attractive material towards solar-driven energy conversion applications: (i) Boron doped $3 \mathrm{C}-\mathrm{SiC}$ as candidate for an intermediate band photovoltaic material, and (ii) 3C-SiC as a photoelectrode for solar-driven water splitting. Absorption spectrum of boron doped $3 \mathrm{C}$-SiC shows a deep energy level at $\sim 0.7 \mathrm{eV}$ above the valence band edge. This indicates that boron doped 3C-SiC may be a good candidate as an intermediate band photovoltaic material, and that bulk like 3C-SiC can have sufficient quality to be a promising electrode for photoelectrochemical water splitting.

\section{Introduction}

Solar energy can provide sufficient power for the global energy demands if it can be efficiently harvested and stored. The photovoltaic cell is the most common device to collect solar energy. The intermediate-band (IB) solar cell, designed by introducing an energy band or level in the semiconductor bandgap, has attracted much attention due to its promising properties to achieve a very high efficiency. The theoretical model for an IB solar cell [1] predicts a maximum efficiency of $63 \%$ in an ideal material having a bandgap at $1.95 \mathrm{eV}$ with an intermediate level at $\sim 0.7 \mathrm{eV}$ above the valence band edge or below the conduction band edge. Cubic silicon carbide ( $3 \mathrm{C}-\mathrm{SiC})$ is a very attractive material for IB solar cells because it has a bandgap of $\sim 2.36 \mathrm{eV}$ and a deep impurity given by the boron acceptor level at $\sim 0.7 \mathrm{eV}$ above the valence band.

Further on, a direct conversion of solar energy into hydrogen from photoelectrochemical (PEC) water splitting provides another attractive, clean and sustainable way to harvest and store solar 
energy in chemical bonds. Hydrogen has a high energy density $(120 \mathrm{~J} / \mathrm{g}$, about three times that of gasoline) and a clean by-product (water) when converted. It is an enormous challenge to find an efficient visible light-absorption semiconductor that can function as an efficient and stable photoelectrode in a PEC cell. In fact, among the commonly used materials, 3C-SiC has a relatively narrow bandgap $(2.36 \mathrm{eV})$ for efficient visible light absorption and proper energetics for water splitting [2].

In this work, we present results in 3C-SiC materials with high structural quality and show their potential a suitable material for solar-driven energy conversion applications.

\section{Experimental details}

High structural quality $3 \mathrm{C}$-SiC material with the thickness of $1 \mathrm{~mm}$ was grown on Si-face 4 degree off-axis $4 \mathrm{H}$-SiC substrates using a sublimation epitaxial growth arrangement [3]. The absorption measurements were done with a PerkinEImer Lambda 950 UV/VIS setup. The undoped $3 \mathrm{C}$-SiC and the boron doped sample (boron concentration of $\sim 10^{18} \mathrm{~cm}^{-3}$ ) were measured after mechanical polishing of the substrate, i.e. free-standing, in order to avoid the disturbance of the absorption from substrates.

Room temperature photoluminescence $(\mathrm{PL})$ were performed on the two boron and nitrogen co-doped 3 C-SiC samples A and B. SIMS results shows that the boron concentration is $2.1 \times 10^{16}$ $\mathrm{cm}^{-3}$ and nitrogen concentration is $7.2 \times 10^{16} \mathrm{~cm}^{-3}$ in sample $A$, while the boron concentration is $4.6 \times 10^{18} \mathrm{~cm}^{-3}$ and nitrogen concentration is $8.5 \times 10^{17} \mathrm{~cm}^{-3}$ in sample $B$. The excitation source was a $406 \mathrm{~nm}$ laser diode and an Ocean Optics Maya2000 Pro spectrometer was used for detection. A long-pass filter with cut-off wavelength of $500 \mathrm{~nm}$ was used to avoid parasitic excitation light to reach the spectrometer.

Photoelectrochemical (PEC) measurements were performed in a three-electrode electrochemical cell with a saturated calomel reference electrode (SCE) and a Pt foil counter electrode in $0.1 \mathrm{M} \mathrm{NaOH}$ electrolyte. The current-potential behavior was measured by Autolab $302 \mathrm{~N}$ with FRA (Eco Chemie, Metrohm). AM 1.5G illumination was provided by $300 \mathrm{~W}$ Xenon Lamp (Newport 67005) with AM 1.5G filter (Newport 81094). The spectrum and intensity of the lamps were calibrated by a spectroradiometer (IL90 International Light) and a laser power meter (Nova II OPHIR) with thermopile sensor, respectively. The PEC measurements were performed using n-type $3 \mathrm{C}$-SiC, $6 \mathrm{H}$-SiC and $4 \mathrm{H}-\mathrm{SiC}$ as photo-anodes. The $6 \mathrm{H}-, 4 \mathrm{H}$-SiC samples are n-type commercial substrates (SiCrystal). They have a nitrogen doping concentration of around $1 \times 10^{18}$ $\mathrm{cm}^{-3}$. The n-type doping in thick 3C-SiC (thickness $\sim 1 \mathrm{~mm}$ ) was estimated around $1 \times 10^{16} \mathrm{~cm}^{-3}$ [3]. Ohmic contacts were fabricated on the backside of the substrate by vacuum evaporation of Al and the contacts were connected to a copper wire.

\section{Results and discussion}

The XRD and low temperature photoluminescence (LTPL) show that 3C-SiC grown by sublimation epitaxy can indeed reach a very high crystal quality. The average FWHM value of XRD omega rocking curves is approximately 40 arcsec [3]. The LTPL emission is only governed by the nitrogen-bound exciton zero-phonon line and its phonon replicas without any defect-related emissions. Moreover, we also observed the multiple bound exciton lines, which are regarded as a characteristic of material having a high structural quality [3].

In order to demonstrate the potential application of $3 \mathrm{C}-\mathrm{SiC}$ as an intermediate band photovoltaic material, we compared the absorption spectra of an undoped and boron doped 3C- 
SiC layers in Fig. 1. The undoped 3C-SiC shows a sharp band-edge absorption (Fig. 1 (a)), which indicates a high crystalline quality as confirmed by XRD and LTPL results. The boron doped 3C-SiC shows a slightly less steep band-edge absorption. This indicates that the doping does not significantly deteriorate the crystalline and the crystal structure quality of boron-doped $3 \mathrm{C}$-SiC is reasonably comparable with that of the undoped sample. In the boron doped 3C-SiC, a broad band around $730 \mathrm{~nm}(1.7 \mathrm{eV})$ can be observed. This absorption band is attributed to the transition between the boron acceptor to the conduction band. From the boron related absorption band, the boron energy level localized at $\sim 0.7 \mathrm{eV}$ above the valence band is estimated.

Figure 1 (b) shows Tauc plots of the undoped and boron doped 3C-SiC, yielding an optical band gap around $2.33 \mathrm{eV}$ for the undoped $3 \mathrm{C}-\mathrm{SiC}$, which is consistent with the bandgap of $2.36 \mathrm{eV}$, as shown in Fig. 1 (b). In the boron doped 3C-SiC, the Tauc plot indicates that the optical band gap is around $2.21 \mathrm{eV}$. This is probably due to the boron doping which results in a less steep band-edge absorption.

(a)

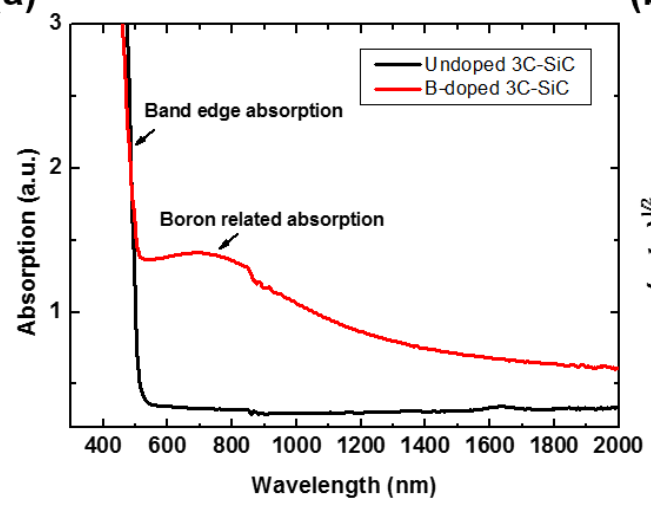

(b)

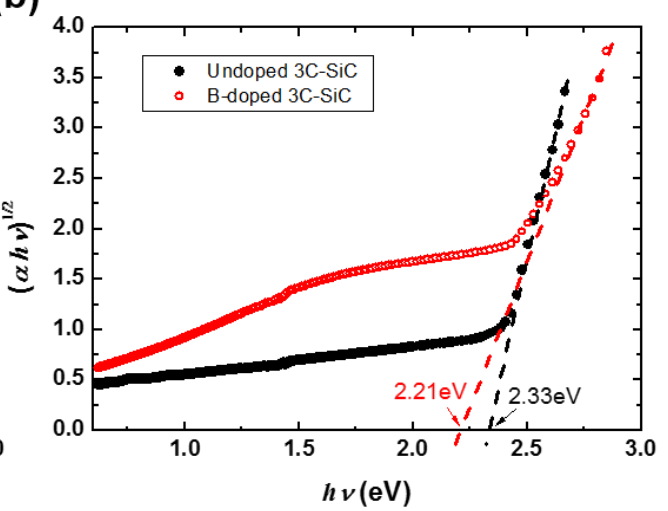

Fig. 1. (a) The absorption spectra of an undoped 3C-SiC and a boron doped 3C-SiC with boron concentration of $\sim 10^{18} \mathrm{~cm}^{-3}$. (b) The optical bandgap plot (Tauc plot) of $(\alpha h u)^{1 / 2} \mathrm{vs}$ photon energy $h u$.

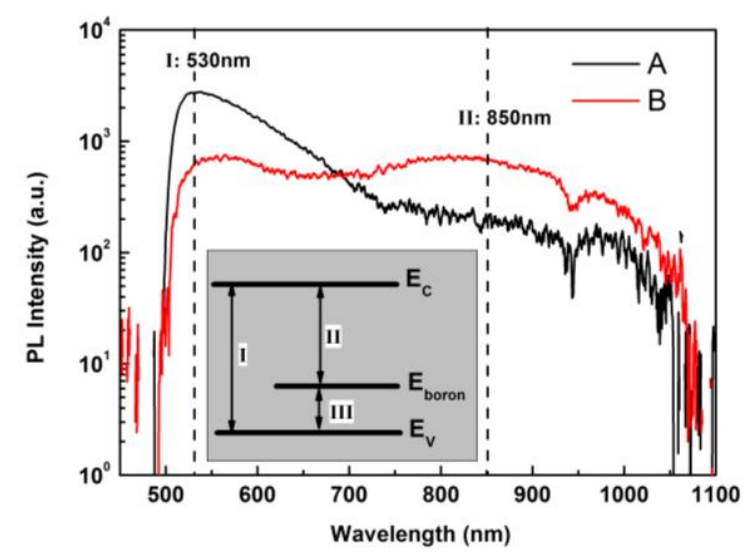

Fig. 2 Room temperature photoluminescence spectra of samples $A$ and $B$ measured at same condition. The inset shows band diagram of the three transitions among the boron energy level ( $\left.E_{\text {boron }}\right)$, conduction band edge $\left(\mathrm{E}_{\mathrm{C}}\right)$ and valence band edge (Ev), illustrating the basic concepts of the intermediate band solar cell. (The artefact at $945 \mathrm{~nm}$ is due to the grating and the PL intensity drops around $1100 \mathrm{~nm}$ due to Si-detector efficiency decrease above $1100 \mathrm{~nm}$ )

To explore the IB solar cell concept in 3C-SiC, we performed room temperature photoluminescence on samples $A$ and $B$, shown in Fig. 2 (note the logarithmic scale). As seen in Fig. 2, both samples show the emission bands centered around $530 \mathrm{~nm}(2.34 \mathrm{eV})$ and a very broad band around at $850 \mathrm{~nm}(1.458 \mathrm{eV})$. The former is very close to the bandgap energy, we attribute it to the near-band-edge emission, corresponding to transition (I) as illustrated in the inset of Fig. 2. It has been demonstrated that the broad emission band around $850 \mathrm{~nm}$ originates from the recombination of electrons in the conduction band with holes bound at the boron acceptors accompanied with its multiple phonon replicas [4]. Thus, we attribute it to transition (II), as 
indicated in the inset of Fig. 2. This assignment can be further supported by the observation that the relative intensities of emission bands I and II are influenced by boron doping. Sample $A$ has higher near-band-edge emission intensity than the free electron to boron acceptor emission intensity while the opposite behavior is observed in sample B. This is reasonable because the increase of boron doping increases the free electron to boron acceptor emission but decreases the near band edge emission. In comparison, the low boron doped sample $A$ has a higher nearband-edge emission relative the free electron to boron acceptor emission. This observation suggests that the emission band at $850 \mathrm{~nm}(1.458 \mathrm{eV})$ may be related to boron impurity level, as observed in the absorption spectrum.

The boron doped 3C-SiC samples show room-temperature radiative recombination of transition (I) and (II). The existence of type (III) emission could not be verified since it falls out of our detection range. This optical activity of boron level indicates that $3 \mathrm{C}-\mathrm{SiC}$ may be a potential candidate as an intermediate bandgap solar cell material.

The 3C-SiC was futher explored as photo-electrode material in the PEC water splitting cells. Fig. 3 shows the currentpotential curves using n-type $3 \mathrm{C}-\mathrm{SiC}, 6 \mathrm{H}-\mathrm{SiC}$ and $4 \mathrm{H}-\mathrm{SiC}$ as photo-anodes. The $3 \mathrm{C}$-SiC anode shows the largest photocurrent density and $6 \mathrm{H}-\mathrm{SiC}$ anode shows a larger photocurrent density than the $4 \mathrm{H}-\mathrm{SiC}$ anodes. This is reasonable considering that the bandgap of $4 \mathrm{H}-\mathrm{SiC}$ is the largest one among all samples and the bandgap of $6 \mathrm{H}$ $\mathrm{SiC}$ is larger than that of $3 \mathrm{C}-\mathrm{SiC}$. The higher photocurrent density of $3 \mathrm{C}$-SiC photoanode may be due to its smaller bandgap of 3C$\mathrm{SiC}(2.36 \mathrm{eV})$.

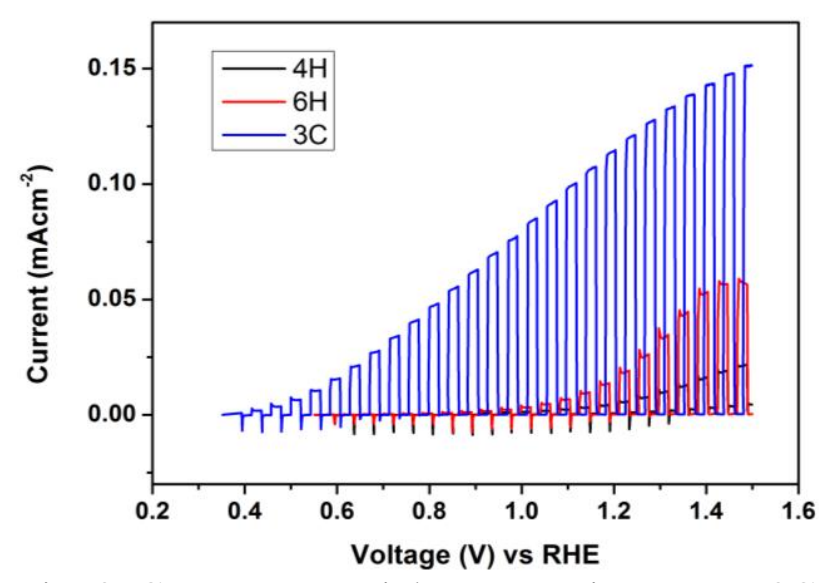

Fig. 3. Current-potential curves using n-type 3C$\mathrm{SiC}, 6 \mathrm{H}-\mathrm{SiC}$ and $4 \mathrm{H}-\mathrm{SiC}$ as photoanodes in $0.1 \mathrm{M}$ $\mathrm{NaOH}$ under chopped AM1.5G illumination.

\section{Summary}

We show that $3 \mathrm{C}-\mathrm{SiC}$ is a promising material for solar-driven energy conversion applications. The absorption and room temperature photoluminescence in boron doped 3C-SiC shows the deep level of $\sim 0.7 \mathrm{eV}$ above the valence band. This indicates that it may be a good candidate as an intermediate band photovoltaic material. We also demonstrate that the high quality $3 \mathrm{C}$-SiC grown by sublimation epitaxy can be applied as an efficient photoelectrode for solar-driven water splitting.

\section{Acknowledgements}

This work was supported by the Swedish Research Council (Vetenskapsrådet, 621-2014-5461; 621-2014-5825), Swedish Energy Agency and the Swedish Governmental Agency for Innovation Systems (Vinnova). IR and AM thank the financial support from the Comunidad de Madrid through the Project MADRID-PV (S2013/MAE-2780).

\section{References}

[1] A. Luque, A. Martí, Physical Review Letters. 78, 5014 (1997).

[2] M. Kato, T. Yasuda, K. Miyake, M. Ichimura, T. Hatayama, Int. J. Hydrogen Energy, 39, 4845 (2014). 
[3] V. Jokubavicius, G. Reza Yazdi, R. Liljedahl, I. G. Ivanov, R. Yakimova, and M. Syväjärvi, Cryst. Growth Des., 14, 6514 (2014).

[4] H. Kuwabara, S. Yamada, physica status solidi (a). 30 (1975) 739-746. 